\title{
Gyropendulum and vibration forces experimental research
}

\author{
Veronica Ivanova, ${ }^{1, *}$, Argen Keremkulov ${ }^{1}$, and Nataliya Kurkan $^{1}$ \\ ${ }^{1}$ National Research Tomsk Polytechnic University, 634050 Tomsk, Russia
}

\begin{abstract}
It is well-known that gyroscope base vibration as usual has a bad influence on accuracy of gyroscopic instruments and systems. During running and testing gyroscopic instruments are exposed to translational vibration, the accuracy and reliability are reducing. With rising demands on the accuracy and reliability of the gyropendulum systems, the problem of finding effects of vibration methods of research base and the possible ways to fix it remains relevant. The purpose of this paper is to present the results of experimental research of vibration influence on the gyropendulum, by using new term "vibration force". Two main regimes were tested: upwards and down displaced centre of mass of gyropendulum.
\end{abstract}

\section{Introduction}

During running gyroscopic instruments are exposed to various disturbances that reduce the accuracy of gyros and can lead to missing of the device efficiency. Angular and translational vibration, vibro-impact and disturbance caused by linear accelerations are mechanical perturbations. Vibration is generated by external and internal sources.

By now, a large number of private tasks of the influence of vibrational perturbations on the gyroscopic devices had been resolved.

The level of external vibration can have frequency from 2 to $10000 \mathrm{~Hz}$, the amplitude up to $1 \mathrm{~mm}$, and vibration overload up to several dozens of $\mathrm{g}$.

Most of all gyroscopic instruments and systems built on the basis of gyropendulums, i.e. the gyroscopes, the center of mass which mismatches with the center of intersection of the axes of suspension are exposed to translational vibration of the base.

\section{Methods and materials}

\subsection{Theoretical background}

The truncated differential equations of motion of gyro pendulum on a vibrating base with down displaced center of mass with the use of the concept of "vibrational force" can be written as [1-7]:

\footnotetext{
* Corresponding author: kurator@tpu.ru
} 


$$
\left.\begin{array}{l}
\dot{\psi}+\left(\frac{m \cdot g \cdot l}{H}+\frac{m \cdot\left(S_{0} \cdot \omega\right)^{2}}{2 \cdot H}\right) \cdot \vartheta=0 \\
-\dot{\vartheta}+\left(\frac{m \cdot g \cdot l}{H}+\frac{m \cdot\left(S_{0} \cdot \omega\right)^{2}}{2 \cdot H}\right) \cdot \psi=0
\end{array}\right\}
$$

Let us analyze the expression in parentheses: $\left(\frac{m \cdot g \cdot l}{H}+\frac{m \cdot\left(S_{0} \cdot \omega\right)^{2}}{2 \cdot H}\right)=Q_{p}$.

The first term $\frac{m \cdot g \cdot 1}{H}=\omega_{0}$ is frequence of gyroscopic wobbling of gyropendulum, appearing because of torque due to gravity $m \cdot g \cdot 1$ and kinetic momentum of gyroscope $H$, the seconf term $\frac{m \cdot\left(S_{0} \cdot \omega\right)^{2}}{2 \cdot H}=\omega_{0 v}$ is angular rate of gyroscopic wobbling appearing because of vibrostabilizing moment $\frac{m \cdot\left(S_{0} \cdot \omega\right)^{2}}{2}$ and kinetic momentum of gyroscope $H$. Hence

$$
Q_{p}=\omega_{0}+\omega_{0 v}
$$

The sum of these two terms (2) is frequence of gyroscopic wobbling of gyropendulum with down displaced center of mass, set on vibration base.

In compact form of general solution will be writtern as

$$
\left.\begin{array}{l}
\vartheta=D \cdot \sin \left[\left(\omega_{0}+\omega_{0 v}\right) \cdot t+\varepsilon\right] \\
\psi=D \cdot \cos \left[\left(\omega_{0}+\omega_{0 v}\right) \cdot t+\varepsilon\right]
\end{array}\right\}
$$

From (3) gyropendelum is undergoing gyroscopic wobbling with amplitude D and frequency $Q_{p}=\omega_{0}+\omega_{0 v}$. We can made a conclusion that the frequence of gyroscopic wobbling $Q_{p}$ has increased in comparison with gyroscope on immovable foundation.

\subsection{The experimental part}

Complex, functional block diagram shown in Figure 1 was used to study gyropendulum with a upwards and down displaced center of mass.

Prototype of gyropendulum 1 was attached to the electrodynamic shaker type table 2 . The table sets harmonic vibration at a fixed frequency. The amplitude and frequency of vibration are given by a signal generator 6 .

Control over the vibration speed of the shaking table was made by piezoelectric sensors 3 , their signals through a matching lock-in amplifier 4 [8-9] were supplied on the programsetting device 5. The last one are monitored and recorded the vibration parameters (amplitude and frequency).

The period of the precessional motion was measured manually using a stopwatch 7 . The level of vibration produced in accordance with the previously performed calculations, based on parameters of real gyropendulum. 


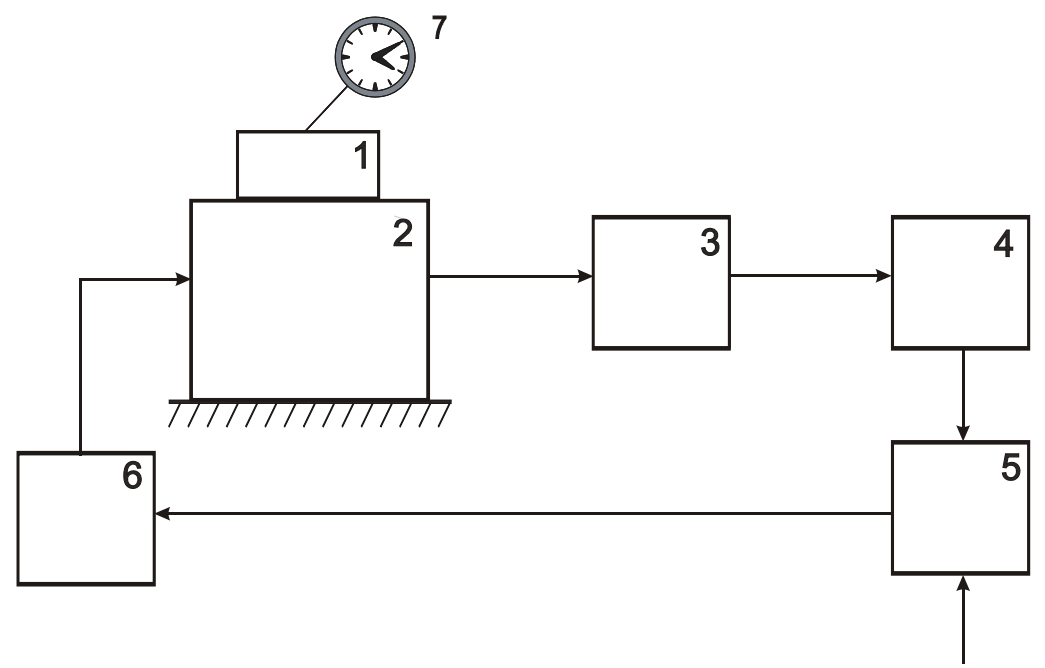

Fig.1. Functional block diagram.

\section{Results and discussion}

The main objective of the experimental studies was qualitative and quantitative evidence of change of speed of gyropendulum precession depending on the vibration parameters of the base $\left(S_{0} ; \omega\right)$.

\subsection{Gyropendulum with an upwards displaced centre of mass}

This is a mode where gyropendulum with an upwards displaced center of mass located above the pivot point, on a vibrating base perfemes a precessional oscillation with the same frequency as gyropendulum with a below the point of suspension to a fixed base was determined with the following vibration parameters: vibration frequency $f=130 \mathrm{~Hz}$; vibration velocity $V=0.9 \mathrm{~m} / \mathrm{s}$; the total precession time of $t=9 \mathrm{~min}$; number of revolutions in time t of the experiment $N=3$.

The period of gyropendulum precession of with a nupwards displaced mass center equals to 3 minutes. The mode in which the precession of gyropendulum absent, i.e. giromatic on a vibrating base acted like astatic gyroscope was fixed. This state of gyropendulum was recorded under the following vibration parameters: vibration amplitude $S_{0}=1.2 \mathrm{~mm}$; circular frequency $\omega=580 \mathrm{rad} / \mathrm{sec}$, i.e. speed of vibration is equal to $V v i b .=0.7$ $\mathrm{m} / \mathrm{s}$.

\subsection{Gyropendulum with a down displaced centre of mass}

Theoretical calculations have shown that the higher the dynamic force acting on gyropendulum is, other words the higher the frequency of vibration of the base (provided that the vibration amplitude constant), the higher the frequency of precession of gyropendulum is [2]. The experiments have confirmed the theoretical calculations. It was founded that the higher the ratio of dynamic forces and gravity ( vibrational force) is, the smaller the period of the precession of gyropendulum is.

The recived results can be used in desing compensation scheme of action of vertical harmonic vibration on the gyropendulum. 


\section{Conclusions}

It was found that the gyroscope with the displaced center of mass, located on a vibrating base, the precession rate depends on the amplitude and frequency $\left(S_{0} ; \omega\right)$ base vibration (i.e, from the dynamic force) and also depends on the center of mass of the gyroscope location (above or below the point of suspension of the gyroscope). Such gyroscope changes its direction depending on the speed value $\left(S_{0} ; \omega\right)$ vibration (i.e. dynamic force compared with the force of gravity). A case where there is no precession (dynamic force balances the force of gravity) was found.

Quantitative changes in the nature of the processional motion of gyropendulum depending on the vibration parameters were confirmed experimentally.

\section{References}

1. K.V. Lineitseva, V.I. Kopytov, The 8th International Scientific and Practical Conference of Students, Post-Graduates and Young Scientists: Modern Technique and Technologies, MTT, 59 (2002)

2. V.S. Volkova, V.I. Kopytov, 5th Korea-Russia International Symposium on Science and Technology, 29 (2001)

3. V.S. Ivanova, 8th International Scientific and Practical Conference of Students, Postgraduates and Young Scientists; "Modem Techniques and Technologies", 42 (2002)

4. E.V. Golodnykh, V.N. Borikov, SIBCON, 6693635 (2013)

5. A.V. Taracenko, V.S. Dmitriev, T.G. Kostuchenko, KORUS, 83 (2004)

6. A.N. Gormakov, KORUS, 20 (2004)

7. V.J. Skorykh, A.N. Gormakov, I.A. Plotnikov, KORUS, 42 (2001)

8. P.F. Baranov, E.I. Tsimbalist, V.N. Borikov, D.G. Soltanova, IMEKO, 116100 (2015)

9. P.F. Baranov, E.I. Tsimbalist, V.E. Baranova, SIBCON, 7146992 (2015) 